\title{
Vegetation and climate from Late Quaternary Thimi Formation (Phaidhoka Section), Bhaktapur, Nepal
}

\author{
Deepak Banjade, Kabir Sharma, and *Khum N. Paudayal \\ Central Department of Geology, Tribhuvan University, Kirtipur, Kathmandu, Nepal \\ (*E-mail: khum99@gmail.com)
}

\begin{abstract}
The Thimi Formation is fluvio-deltaic deposit that constitutes the uppermost part of the sedimentary sequence in the Kathmandu Basin, and is featured by carbonaceous and diatomaceous clay, silty clay, silt, fine to medium grained sand beds, and thin to medium lignite beds. The Phaidhoka Section is located on the way to Nala from Chyamasingh, and is one of the major exposures of the Thimi Formation. Forty four samples were collected from $25 \mathrm{~m}$ thick surface exposure for palynological study. The study revealed the dominance of gymnosperm over the angiosperm and herbaceous members. The pollen diagram suggested Pinus, Picea and Quercus as the most dominant trees whereas Poaceae is other dominant among the grasses. Three major pollen assemblage zones were marked in the Thimi Formation. Zone P-I indicated warm temperate climate, whereas zone P-II and P-III indicated cold temperate climate. Molluscan operculum in the upper part indicated shallow water condition. The Bovid molars, limb and pelvic bones from the middle part of the section confirm the early findings of molar bones in this area.
\end{abstract}

Keywords: Late Quaternary, palynology, climate, Thimi Formation, Kathmandu Basin Paper Received: 11 July 2015

Paper Accepted: 12 December 2015

\section{INTRODUCTION}

The Kathmandu Basin is an intermontane basin filled with very thick sequence of lacustrine and fluvial deposits of Plio-Pleistocene age (Yoshida and Igarashi 1984, Moribayashi and Mauro 1980). The oldest formation is known as the Lukundol Formation exposed to the southern part of the basin. The primary basin was mainly confined to the southern part of the valley and was relatively small compared to the later. It started receiving sediments from the southern part and the Lukundol Formation and was formed in the process (Kizaki 1994). But, the uplifting of the Mahabharat Range in the southern part changed the course and the lake started shifting towards north. The tilting of the beds of the Lukundol Formation to the north is one of the major evidence. The new lake was much large and covered large area resulting in the deposition of younger sediments named as the Gokarna, Thimi and Patan formations (Fig. 1). The lake completely drained at the end of the last glacial epoch (Yoshida and Igarashi 1984). The Thimi Formation, a fluvio-lacustrine deposit, is distributed around Pashupatinath, Tribhuvan International Airport, Thimi, Bhaktapur and Patan comprises alternating layers of carbonaceous and diatomaceous clay, silty clay, silt and fine to coarse sand.

Yoshida and Igarashi (1984), Igarashi et al. (1988), and Yoshida and Gautam (1988) divided the basin sediments into the older-stage deposits (i.e. the Lukundol Formation), middlestage deposits (i.e. the Boregaon, Chapagaon and Pyanggaon terrace deposits), and younger-stage deposits (i.e. the Gokarna, Thimi and Patan formations). A number of palynological investigations were also carried out by many researchers from the different sections of the Kathmandu Basin sediments (Vishnu- Mittre and Sharma 1984, Fuji and Sakai 2001, 2002, Paudayal and Ferguson 2004, Paudayal 2005, Paudayal 2006, Bhandari and Paudayal 2007, Paudayal 2011a, Paudayal 2011b).

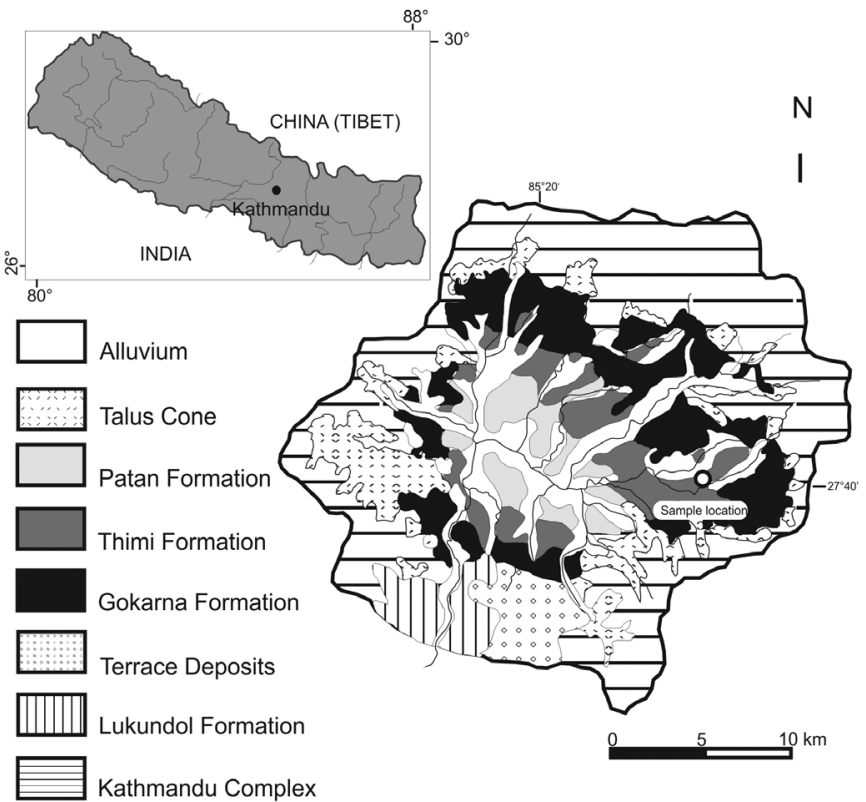

Fig 1: Geological map of the study area showing sampling location (black open cirlce) at Phaidhoka, Bhaktapur. 


\section{MATERIALS AND METHODS}

Samples were taken from $25 \mathrm{~m}$ thick surface exposure at Phaidhoka, Bhaktapur belonging to the Thimi Formation (Fig. 2). The sediments of the Thimi Formation are relatively finer indicating the deposition might have occurred from suspension settling and only limited bedload transport via weak currents. The thin, parallel laminations of alternating silt and silty clay indicate widespread deposition from suspension over the sand beds. The sand and silt layer consists of cross bedded structure; wave ripple laminated structure and sometimes with the distortion of ripples in sand beds. The flow structure and distorted cross beds suggest the liquefaction or fludisation and hydro-plastic deformation. The deformation was considered to have been caused by paleo-earthquakes during sedimentation (Gajurel et al. 1998).

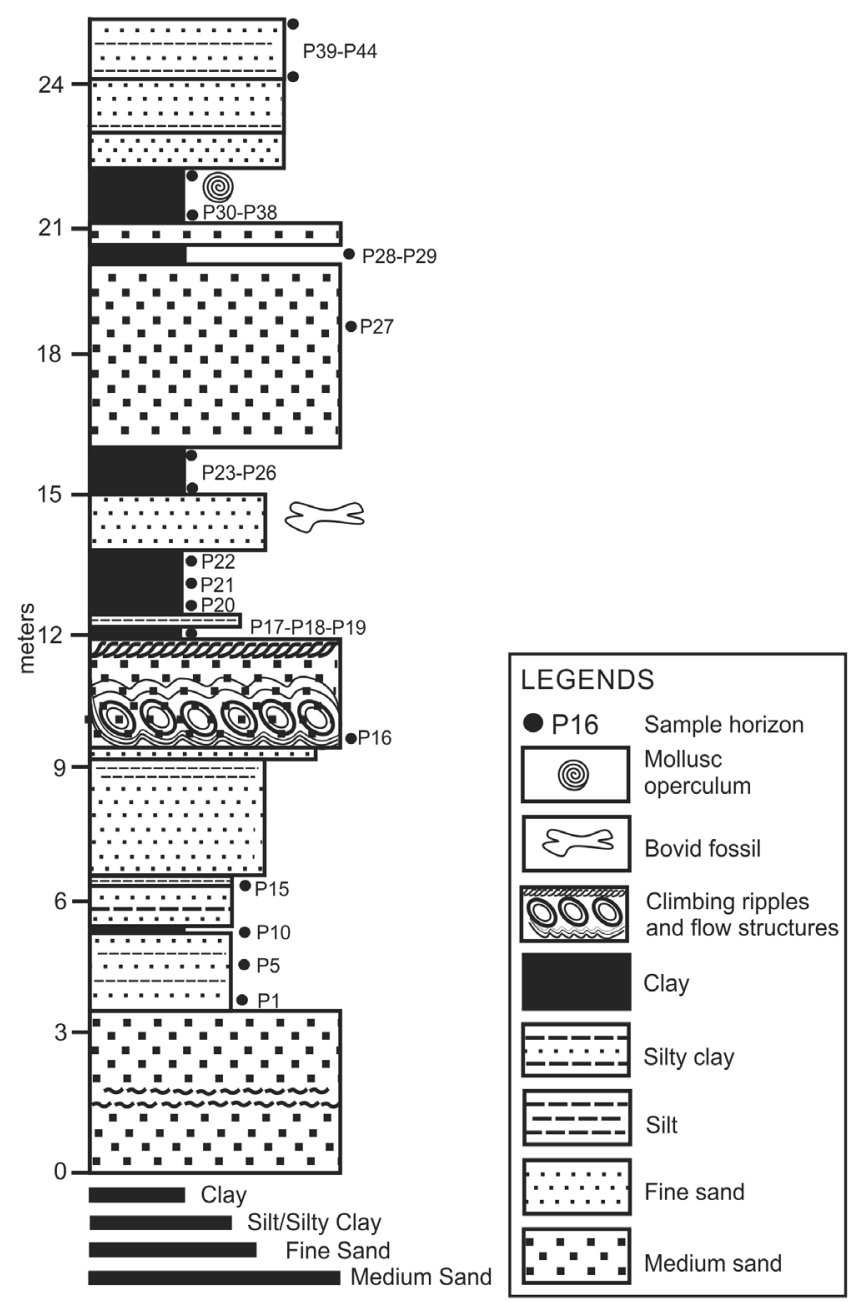

Fig. 2: Lithology of the Thimi Formation, Phaidhoka section, Bhaktapur.

The palynological samples were prepared at the Central Department of Geology, Tribhuvan University, Kirtipur, Kathmandu, following the method described by Zetter (1989) and Ferguson et al. (2007). This includes series of chemical treatment of sediments such as concentrated $\mathrm{HCl}, \mathrm{HF}$,
$\mathrm{CH}_{3} \mathrm{COOH}, \mathrm{KClO}_{3},\left(\mathrm{CH}_{3} \mathrm{CO}\right)_{2} \mathrm{O}, \mathrm{H}_{2} \mathrm{SO}_{4}$. The organic content was separated using heavy liquid $\mathrm{ZnBr}_{2}$ having specific gravity $2 \mathrm{gm} / \mathrm{cm}^{3}$. The extracted organic material was then preserved in glycerin for microscopy. At least 300 pollen grains from each sample were point-counted under an OLYMPUS BX-43 light microscope. The palynomorphs were identified with the help of various pollen atlases and published literatures. The TILIA software was used for statistical analysis and preparing a pollen diagram.

\section{RESULTS}

\section{a. Invertebrate fossils}

In the upper part of the section a clay layer full of molluscan opercula are discovered. They are identified under family Bithyniidae belonging to the genus Digoniostoma and Gabbia, respectively.

\section{b. Vertebrate fossils}

Six large molars along with fragmented limb and pelvic bones belonging to family Bovidae (possibly Bos or Bubalus) are recorded from the middle part of the section. The molars and bones are still under investigation to their lower taxonomic level. The vertebrate fossils will be dealt in a separate paper.

\section{c. Plant microfossils}

Significant amount of plant microfossils (palynomorphs) helped us to understand the paleoclimatic evolution of the study area. Out of forty four samples collected from the Phaidhoka Section, twenty two samples were rich in palynomorphs. Altogether 94 species of plants belonging to 74 genera and 40 families were identified (Plates I-IX). The plant microfossils obtained from this section are identified and described below.

\section{i. Gymnosperms}

\section{Family: Pinaceae}

Cedrus sp. (Plate I, Fig. 1)

Shape: Bisaccate; Size: $70 \mu \mathrm{m}$, corpus $50 \mu \mathrm{m}$, sacci $20 \mu \mathrm{m}$; corpus perforate; Exine is thick, corpus attached with lower half of the sacci.

Picea sp. (Plate I, Fig. 2)

Shape: Bisaccate; Size: $70 \mu \mathrm{m}$, corpus $50 \mu \mathrm{m}$, sacci $20 \mu \mathrm{m}$; corpus perforate rugulate, alveolate, attached with majority width of corpus, weak angle between sacci and corpus.

\section{Pinus roxburghii (Plate I, Figs. 3-4)}

Shape: Bisaccate; Size: $80 \mu \mathrm{m}$, corpus $50 \mu \mathrm{m}$, sacci $35 \mu \mathrm{m}$; corpus rugulate, the attachment of corpus and sacci is featured by distinct angle, sacci attached with majority width of corpus, polar view shows the attachment of corpus exists at upper half.

\section{Pinus wallichiana (Plate I, Figs. 5-6)}

Shape: Bissacate; Size: $80 \mu \mathrm{m}$, corpus $50 \mu \mathrm{m}$, sacci $30 \mu \mathrm{m}$; 


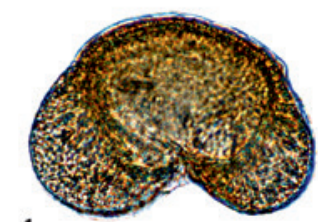

1.

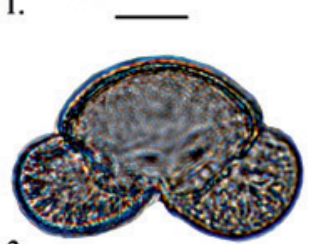

3.

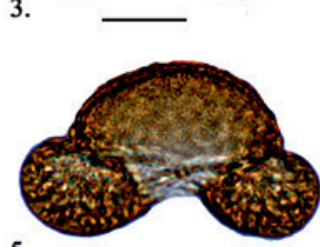

5.

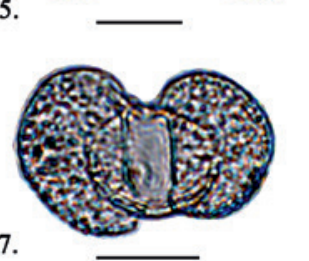

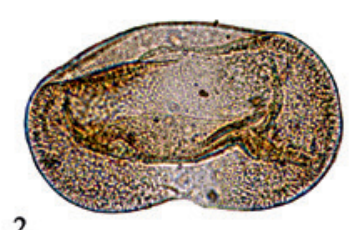

2 .

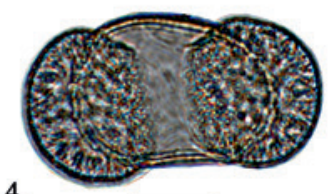

4.

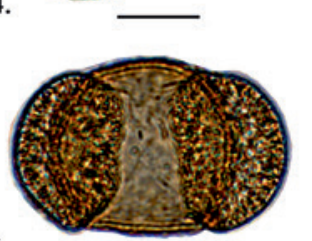

6.

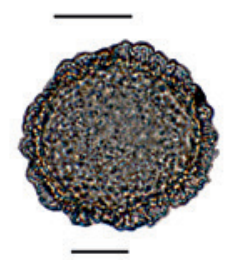

9.

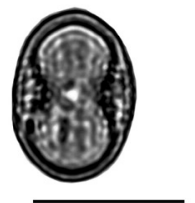

12.

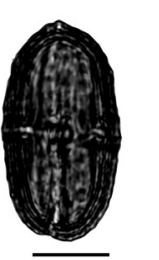

15.

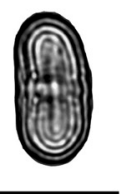

18.

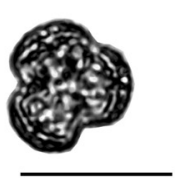

10.

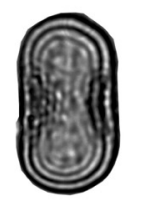

13.

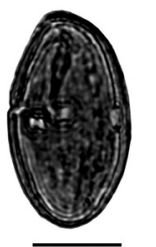

16.

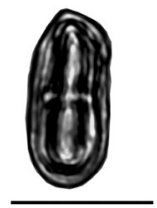

19.

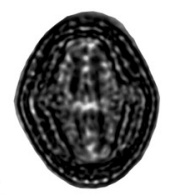

11.

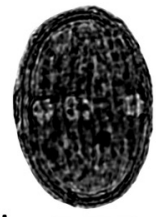

14.

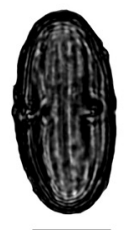

17.

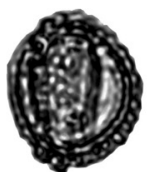

20.

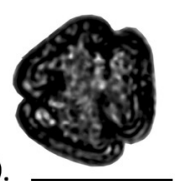

Plate-I: 1- Cedrus sp., 2- Picea sp., 3 and 4- Pinus roxburghii (equatorial and polar view), 5 and 6- Pinus Wallichiana (Equatorial and Polar view), 7- Podocarpus sp., 8- Tsuga sp. (scale bar $=20 \mu \mathrm{m})$.

corpus is rugulate and rough, sacci finely alveolate, attached with majority width of corpus, polar view shows the attachment of corpus with sacci existing at upper half, corpus making distinct angle with sacci.

\section{Tsuga sp. (Plate I, Fig. 8)}

Shape: Monosaccate; Size: $70 \mu \mathrm{m}$, circular in polar view, corpus echinate and foveolate, verrucate to regulate, sacci echinate and foveolate.

\section{Family: Podocarpaceae}

\section{Podocarpus sp. (Plate I, Fig. 7)}

Shape: Bisaccate; Size: $50 \mu \mathrm{m}$, corpus $30 \mu \mathrm{m}$, saci $20 \mu \mathrm{m}$; corpus finely verrucate, sacci finely alveolate, attached with majority width of corpus, size of the corpus smaller than sacci.

\section{ii. Angiosperms (Dicotyledons)}

\section{Family: Acanthaceae}

\section{Justicia sp. (1) (Plate II, Fig. 9)}

Shape: Prolate; Size: polar axis $20 \mu \mathrm{m}$, equatorial axis $15 \mu \mathrm{m}$;. Aperture: colporate. Exine: $1.5 \mu \mathrm{m}$, sexine and nexine having equal thickness, sexine regulate and perforate around aperture.

Plate-II: 9. Justicia sp. 1, 10. Justicia sp. 2, 11. Strobilanthes sp. 1, 12. Strolbilanthes sp. 2, 13. Strobilanthes sp. 3, 14. Strobilanthes sp. 4, 15. Apiaceae gen. indet.1, 16. Apiaceae gen. indet. 2, 17-18 Ilex sp., 19-20. Araliaceae gen. indet. (scale bar $=20 \mu \mathrm{m})$.

\section{Justicia sp. (2) (Plate II, Fig. 10)}

Shape: Prolate; Size: polar axis $25 \mu \mathrm{m}$, equatorial axis 15 $\mu \mathrm{m}$; Aperture: Colporate; Exine: $1.6 \mu \mathrm{m}$, sexine thicker than nexine, nexine alveolate and rough near aperture.

Strobilanthes sp. (1) (Plate II, Fig. 11)

Shape: Prolate; Size: Polar axis $60 \mu \mathrm{m}$, equatorial axis 40 $\mu \mathrm{m}$; Aperture: Tri-colporate; Exine: $5 \mu \mathrm{m}$, sexine thicker than nexine, sexine coarsely reticulate.

Strobilanthes sp. (2) (Plate II, Fig. 12)

Shape: Prolate; Size: Polar axis $60 \mu \mathrm{m}$, equatorial axis $30 \mu \mathrm{m}$; Aperture: Tri-colporate, Exine: $5 \mu \mathrm{m}$, Sexine thicker than nexine, sexine divided into longitudinal strips and coarsely reticulate.

\section{Strobilanthes sp. (3) (Plate II, Fig. 13)}

Shape: Prolate; Size: Polar axis $50 \mu \mathrm{m}$, equatorial axis $25 \mu \mathrm{m}$; Aperture: Tri-colporate; Exine: $3.5 \mu \mathrm{m}$, sexine and nexine having equal thickness, sexine around aperture regulate and granulate.

\section{Strobilanthes sp. (4) (Plate II, Fig. 14)}

Shape: Prolate; Size: Polar axis $60 \mu \mathrm{m}$, equatorial axis 30 $\mu \mathrm{m}$; Aperture: Tri-colporate, Exine: $5 \mu \mathrm{m}$, sexine thicker than 
nexine, sexine further subdivided into longitudinal strips, sexine near aperture foveolate.

\section{Family: Apiaceae}

Apiaceae gen. indet. (1) (Plate II, Fig. 15)

Shape: Prolate; Size: Polar axis 20-23 $\mu \mathrm{m}$, equatorial axis 10$12 \mu \mathrm{m}$; Aperture: Tri-colporate, colpi extended almost upto poles; Exine: $1.3 \mu \mathrm{m}$, regulate and granulate.

Apiaceae gen. indet. (2) (Plate II, Fig. 16)

Shape: Prolate; Size: Polar axis $25 \mu \mathrm{m}$, equatorial axis $15 \mu \mathrm{m}$; Aperture: Tri-colporate; Exine: $2.3 \mu \mathrm{m}$, nexine thicker than sexine, sexine regulate and granulate.

\section{Family: Araliceae}

Araliaceae gen indet. (Plate II, Fig. 17-18)

Shape: Spheroidal and sub-prolate; Size: Equatorial view 25 $\mu \mathrm{m}$, Polar view $20 \mu \mathrm{m}$; Aperture: Coloporate; Exine: $4.5 \mu \mathrm{m}$, reticulate, regulate and granular.

\section{Family: Balsaminaceae}

Impatiens sp. (Plate III, Fig. 21)

Shape: Oblate, rectangular; Size: Equatorial axis $32 \mu \mathrm{m}$; Aperture: Tetra-colporate; Exine: $1.8 \mu \mathrm{m}$, thickness of sexine and nexine similar, reticulate and granulate.

\section{Family: Betulaceae}

Alnus sp. (Plate III, Fig. 22)

Shape: Oblate, pentangular in polar view; Size: 18-20 $\mu \mathrm{m}$; Aperture: Pentaporate, post vestibulum type; Exine: $1.5 \mu \mathrm{m}$, sexine thicker than nexine, tectum rugulate and micro-echinate.

\section{Betula sp. (Plate III, Fig. 23)}

Shape: Oblate, triangular in polar view; Size: $20 \mu \mathrm{m}$; Aperture: Tri-porate, circular pores, pores with chamber separates inner and outer walls; Exine $2.4 \mu \mathrm{m}$, sexine scrabate to regulate and thick at apex.

\section{Corylus sp. (Plate III, Fig. 24)}

Shape: Oblate, triangular in polar view; Size: $24 \mu \mathrm{m}$; Aperture: Tri-porate; Exine: $3.4 \mu \mathrm{m}$, exine has no arci and does not thicken in the aperture region. The sculpture of exine is slightly rugulate. Nexine thickness is not separated from the foot-layer of the aperture region.

\section{Family: Bombacaceae}

\section{Bombax sp. (Plate III, Fig. 25)}

Shape: Oblate, triangular in polar view; Size: $35 \mu \mathrm{m}$; Aperture: Tri-colporate, situated more or less at mid points; Exine: 3.3 $\mu \mathrm{m}$, nexine thicker than sexine, clearly stratified, rugulate, alveolate, exine scrabate and reticulated.

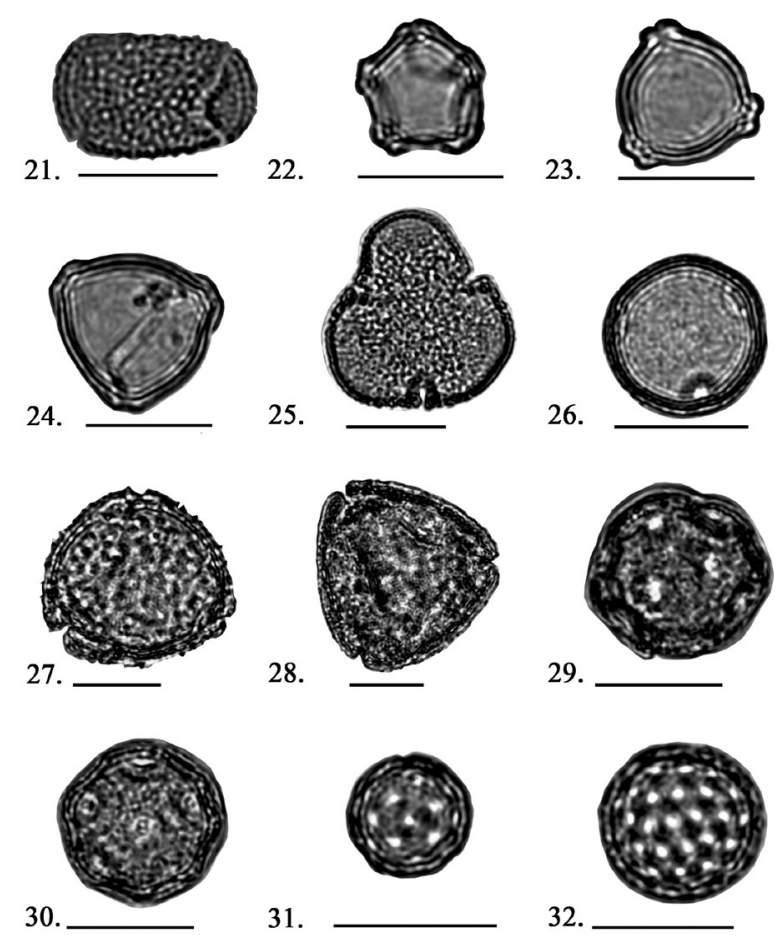

Plate-III: 21. Impatiens sp. 22. Alnus sp. 23. Betula sp. (Family Betulaceae), 24. Corylus sp. 25. Bombax sp. 26. Campanulaceae gen. indet. 27. Lonicera sp. (1), 28. Lonicera sp. (2), 29. Caryophyllaceae gen. indet. (1), 30. Caryophyllaceae gen. indet. (2), 31. Chenopodiaceae gen. indet. (1), 32. Chenopodiaceae gen. indet. (2) (scale bar $=20 \mu \mathrm{m})$.

\section{Family: Campanulaceae}

Campanulaceae gen. indet. (Plate III, Fig. 26)

Shape: Prolate, circular in polar view; Size: $25 \mu \mathrm{m}$; Aperture: Tri-porate, Exine $3.4 \mu \mathrm{m}$, sexine thicker than nexine, rugulate with low relief reticulum.

\section{Family: Caprifoliaceae}

\section{Lonicera sp. (1) (Plate III, Fig. 27)}

Shape: More or less spheroidal, oblate; Size: 40-44 $\mu \mathrm{m}$; Aperture: Tri-colporate, colpi short; Exine: $4 \mu \mathrm{m}$, exine scabrous, echinate or micro-echinate, rugulate, foveolate.

\section{Lonicera sp. (2) (Plate III, Fig. 28)}

Shape: Oblate, triangular in polar view; Size: $45-47 \mu \mathrm{m}$; Aperture: Tri-colporate; Exine: $4 \mu \mathrm{m}$, sexine is thicker than nexine, rough and foveolate, echinate to microechinate.

\section{Family: Caryophyllaceae}

Caryophyllaceae gen. indet. (1) (Plate III, Fig. 29)

Shape: Spheroidal; Size: 28-30 $\mu \mathrm{m}$; Aperture: Pantoporate; Exine: $2 \mu \mathrm{m}$, thickness between sexine and nexine similar, perforated rugulate and spinulated sexine. 
Caryophyllaceae gen. indet. (2) (Plate III, Fig. 30)

Shape: Spheroidal or globular; Size: 22-25 $\mu \mathrm{m}$; Aperture: Pantoporate; Exine: $4 \mu \mathrm{m}$, sexine thicker than nexine, sexine perforated, rugulate, unevenly reticulated.

\section{Family: Chenopodiceae}

Chenopodiaceae gen. indet. (1) (Plate III, Fig. 31)

Shape: Spheroidal or globular; Size: 12-14 $\mu \mathrm{m}$; Aperture: Pantoporate, pore circular; Exine: $2 \mu \mathrm{m}$, Sexine thicker than nexine, sexine spinulated, perforate, pore membrane granulate, spinulate.

Chenopodiaceae gen. indet. (2) (Plate III, Fig. 32)

Shape: Spheroidal; Size: 25 m; Aperture: Pantoporate; Exine: $3 \mu \mathrm{m}$, sexine thicker than nexine.

\section{Family: Compositae}

\section{Artemisia sp. (1) (Plate IV, Fig. 33)}

Shape: Prolate, lobate, circular in polar view; Size: Polar axis 25-27 $\mu \mathrm{m}$; Aperture: Tricolporate; Exine: $3 \mu \mathrm{m}$, sexine is thicker than nexine, clearly stratified, sexine in meso-colpium thicker than in the colpi area.

33.

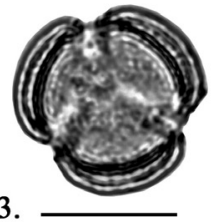

36.

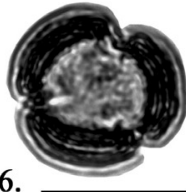

39.

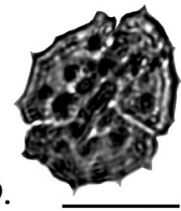

42.

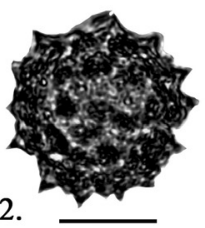

34.

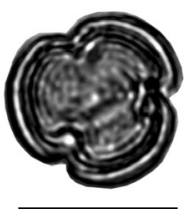

35.
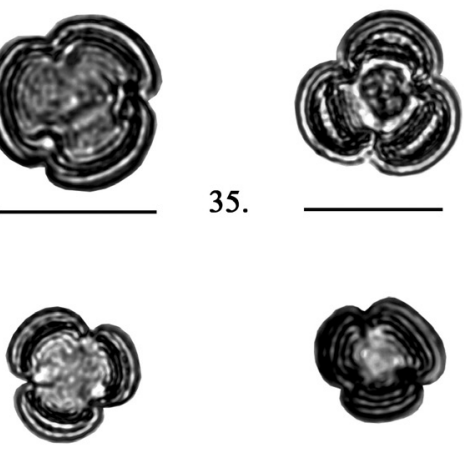

37.

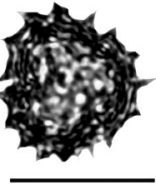

43.

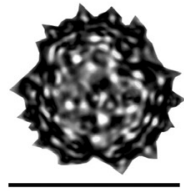

Artemisia sp. (2) (Plate IV, Fig. 34)

Shape: Prolate, lobate, circular in polar view; Size: Polar axis 22-25 $\mu \mathrm{m}$; Aperture: Tricolporate, colpi as long as polar axis; Exine: $4 \mu \mathrm{m}$, sexine thicker than nexine, both distinctly stratified, varying sexine thickness.

Artemisia sp. (3) (Plate IV, Fig. 35)

Shape: Prolate, lobate, circular in polar view; Size: $20-22 \mu \mathrm{m}$; Aperture: Tricolporate, reduced colpi; Exine: $3 \mu \mathrm{m}$, sexine much thicker than nexine.

Artemisia sp. (4) (Plate IV, Fig. 36)

Shape: Prolate, lobate, circular in polar view; Size: $25 \mu \mathrm{m}$; Aperture: Tri-colporate, colpi wide at equator and narrow at poles, distinct with thick margins; Exine: $2 \mu \mathrm{m}$, sexine and nexine similar in thickness, micro-echinate and granulated.

Artemisia sp. (5) (Plate IV, Fig. 37)

Shape: Prolate, lobate, circular in polar view; Size: 15-17 $\mu \mathrm{m}$; Aperture: Tri-colporate, colpi as wide as polar axis; Exine: $1 \mu \mathrm{m}$, sexine and nexine clearly stratified, micro-echinate, granulated

\section{Artemisia sp. (6) (Plate IV, Fig. 38)}

Shape: Prolate, lobate, semi-circular in polar view; Size: 10$12 \mu \mathrm{m}$; Aperture: Tri-colporate, reduced and contracted colpi; Exine: $2 \mu \mathrm{m}$, sexine thicker than nexine, distinctly stratified ornamentation.

\section{Compositae gen. indet. (1) (Plate IV, Fig. 39)}

Shape: Sub-prolate; Size: $32 \mu \mathrm{m}$; Aperture: Tri-colporate; Exine: $6 \mu \mathrm{m}$, sexine thicker than nexine, echinated and perforated, surface granulated, spines $2 \mu \mathrm{m}$ in length.

Compositae gen. indet. (2) (Plate IV, Fig. 40)

Shape: Prolate; Size: $20 \mu \mathrm{m}$; Aperture: Tri-colporate; Exine: $4 \mu \mathrm{m}$, sexine is thicker than nexine, echinated, perforated, granulated.

\section{Compositae gen. indet. (3) (Plate IV, Fig. 41)}

Shape: Prolate, circular in polar view; Size: 22-25 $\mu \mathrm{m}$; Aperture: Tri-colporate; Exine: $5 \mu \mathrm{m}$, exine straitified, exine is thick and coarsely granular, echinated, reduced spines.

Compositae gen. indet. (4) (Plate IV, Fig. 42)

Shape: Prolate, circular in polar view; Size: $40 \mu \mathrm{m}$; Aperture: Tri-colporate; Exine: $2 \mu \mathrm{m}$, sexine thicker, echinated, coarsely granular, spines $2 \mu \mathrm{m}$ in length.

Compositae gen. indet. (5) (Plate IV, Fig. 43)

Shape: Prolate, circular in polar view; Size: $16 \mu \mathrm{m}$; Aperture: Tricolporate, colpuscovered with granular elements, narrow, smooth and sunken colpi; Exine: $4 \mu \mathrm{m}$, roughly granulated, echinated, thicker sexine. 
Compositae gen. indet. (6) (Plate IV, Fig. 44)

Shape: Sub-prolate, hexagonal in polar view; Size: 33-35 $\mu$ m; Aperture: Tri-coloporate.

Family: Dipsacaceae

Dipsacus sp. (1) (Plate V, Fig. 45)

Shape: Semi-oblate, spheroidal; Size: $65 \mu \mathrm{m}$; Aperture: Tricolporate; Exine: $6 \mu \mathrm{m}$, very thicker sexine which is stratified into two layers, sexine echinate and microechinate, perforated and granulated, varying spine size, larger spines are stupashaped.

Dipsacus sp. (2) (Plate V, Fig. 46)

Shape: Oblate, spheroidal; Size: 55-60 $\mu \mathrm{m}$; Aperture: Tricolporate; Exine: $7 \mu \mathrm{m}$, sexine is thicker, extended and divided, granulated, sexine stratified into two layers, echinate and microechinate.

\section{Family: Elaeagnaceae}

Elaeagnus sp. (Plate V, Fig. 47)
45.

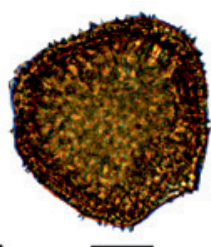

48.

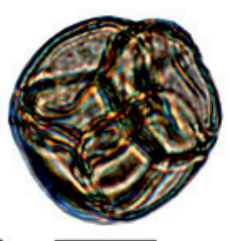

51.

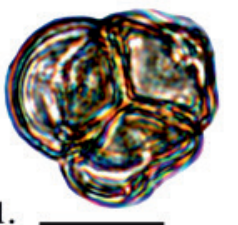

54.

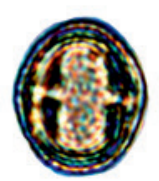

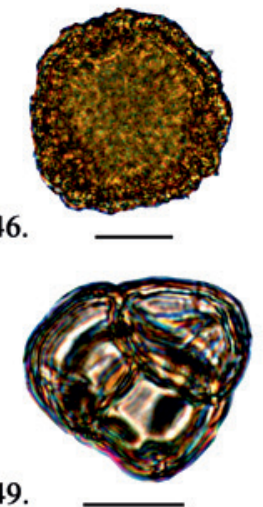

49.

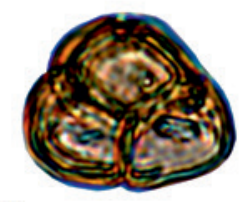

52.

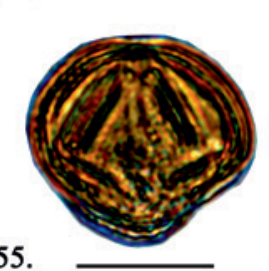

47.

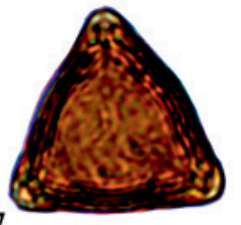

50.
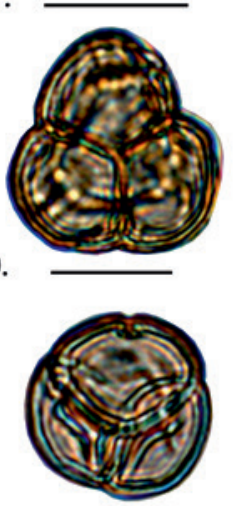

53.

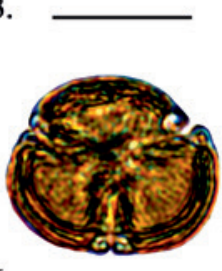

56.
Shape: Oblate, triangular in polar view; Size: $25-27 \mu \mathrm{m}$; Aperture: Tri-colporate; Exine: $4 \mu \mathrm{m}$, sexine thinner than nexine, surface granulate and slightly undulating.

\section{Family: Ericaceae}

Rhododendron sp. (1) (Plate V, Fig 48)

Shape: Tetrahedral tetrad; Size: $40 \mu \mathrm{m}$; Aperture: Tri-colporate; Exine: $3 \mu \mathrm{m}$, sexine and nexine similar in thickness, probably psilate.

\section{Rhododendron sp. (2) (Plate V, Fig. 49)}

Shape: Tetrahedral tetrad; Size: $38 \mu \mathrm{m}$; Aperture: Tri-colporate; Exine $5 \mu \mathrm{m}$, sexine thicker than nexine.

Rhododendron sp. (3) (Plate V, Fig. 50)

Shape: Tetrahedral tetrad; Size: $40 \mu \mathrm{m}$; Aperture: Tri-colporate; Exine: $5 \mu \mathrm{m}$, sexine thicker than nexine.

Rhododendron sp. (4) (Plate V, Fig. 51)

Shape: Tetrahedral tetrad; Size: 35-38 $\mu$; Aperture: Tricolporate; Exine: $6 \mu \mathrm{m}$, thickness of exine not similar, undulating, sexine thicker than nexine.

Ericaceae gen. indet. (1) (Plate V, Fig. 52)

Shape: Tetrahedral tetrads; Size: $25-28 \mu \mathrm{m}$; Aperture: Tricolporate; Exine: $3.5 \mu \mathrm{m}$, sexine and nexine similar in thickness.

Ericaceae gen. indet. (2) (Plate V, Fig. 53)

Shape: Tetrahedral tetrad; Size: 20-22 $\mu \mathrm{m}$; Aperture: Tricolporate; Exine: $3.5 \mu \mathrm{m}$; sexine much thicker than nexine.

\section{Family: Euphorbiaceae}

Euphorbiaceae gen. indet. (1) (Plate V, Fig. 54)

Shape: Prolate, semicircular in polar view; Size: $17 \mu \mathrm{m}$; Exine: $3 \mu \mathrm{m}$, narrow, long colpus, marginal zone of colpus smooth, exine structure reticulate-rugulose.

Euphorbiaceae gen. indet. (2) (Plate V, Fig. 55-56)

Shape: Semi-circular in equatorial view, circular in polar view; Size: Equatorial axis $3 \mu \mathrm{m}$, Polar axis $32 \mu \mathrm{m}$; Exine: $6.5 \mu \mathrm{m}$, reticulated exine, sexine thicker than nexine, long narrow colpus.

\section{Family: Fagaceae}

\section{Castanopsis sp. (Plate VI, Fig. 57)}

Shape: Prolate and circular in equatorial view; Size: $14 \mu \mathrm{m}$; Aperture: Tri-colporate, colpi long and narrow; Exine: $2 \mu \mathrm{m}$, sexine and nexine similar in thickness, radially symmetrical, sculpture tangled-stringy-rugulose.

Quercus sp. (Plate VI, Fig. 58-59)

Shape: Prolate in equatorial view and lobate in polar view; 


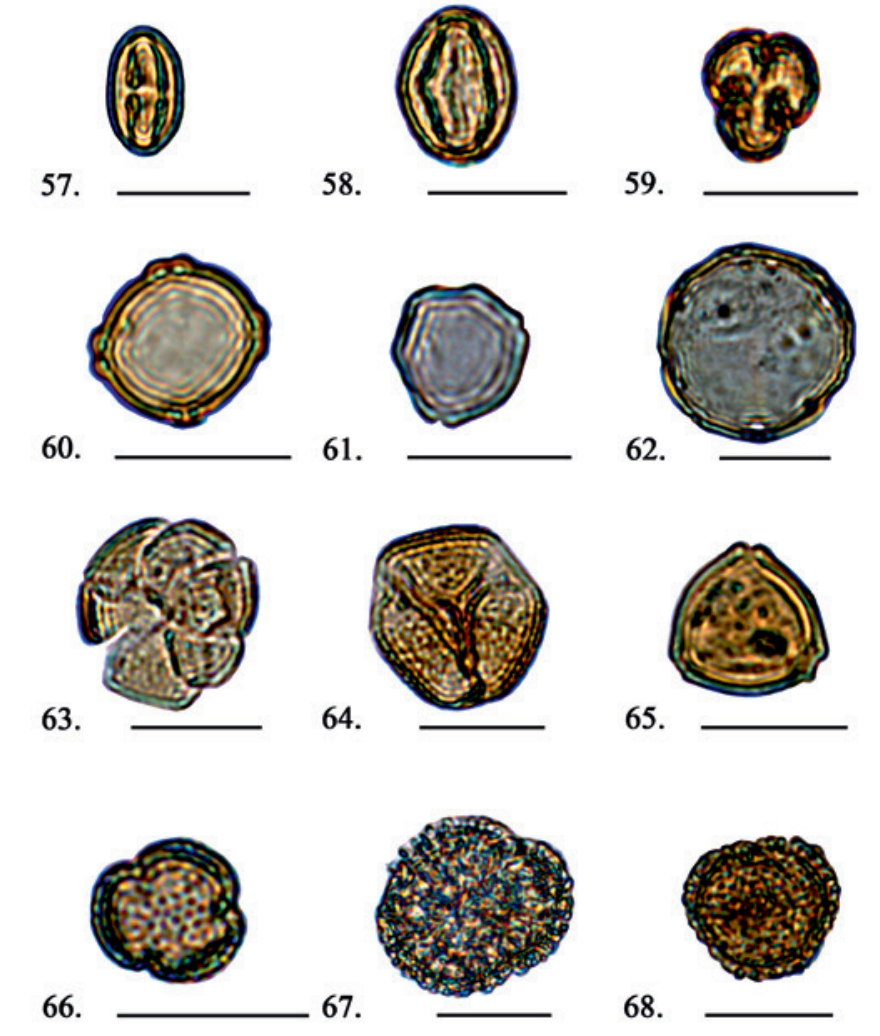

Plate-VI: 57. Castanopsis sp. (Family Fagaceae), 58-59. Quercus sp., Equatorial and Polar view, 60. Myriophyllum sp., 61. Engelhardtia sp. (Family Juglandaceae), 62. Juglans sp., 63. Family Labiatae gen. indet., 64. Nymphoides sp. 65. Myrica sp. 66. Fraxinus sp., 67. Jasminum sp., 68. Ligustrum sp. (scale bar $=20 \mu \mathrm{m})$.

Size: Equatorial axis $18 \mu \mathrm{m}$, polar axis $15 \mu \mathrm{m}$; Aperture: Tri-colporate, long narrow colpi; Exine: mesocolpium $2 \mu \mathrm{m}$ relatively short, straight furrows, occasionally bent in the pore areas, surface verrucate to scabrate with elements of various sizes.

\section{Family: Haloragaceae}

Myriophyllum sp. (Plate VI, Fig. 60)

Shape: Oblate, semi-circular in equatorial view; Size: Equatorial axis $20 \mu \mathrm{m}$; Aperture: Pentaporate; Sexine slightly thicker than nexine, granulated.

\section{Family: Juglandaceae}

\section{Engelhardtia sp. (Plate VI, Fig. 61)}

Shape: Oblate, triangular in outline; Size: $16 \mu \mathrm{m}$; Aperture: Triporate; Exine: $3 \mu \mathrm{m}$, sexine thicker than nexine, granulated.

\section{Juglans sp. (Plate VI, Fig. 62)}

Shape: Oblate, spheroidal to sub-spheroidal, circular; Size: 38 $\mu \mathrm{m}$; Aperture: Pantoporate; Exine: $2.5 \mu \mathrm{m}$, sexine thicker than nexine, tectum microechinate.

\section{Family: Labiatae}

Labiatae gen. indet. (Plate VI, Fig. 63)

Shape: Oblate to sub-spheroidal; Size: $28 \mu \mathrm{m}$; Aperture: Hexacolpate; Exine: $3 \mu \mathrm{m}$, sexine slightly thicker nexine, smoothly reticulated, furrows are generally straight and narrow.

\section{Family: Menispermaceae}

Nymphoides sp. (Plate VI, Fig. 64)

Shape: Triangular in polar view; Size: $28-30 \mu \mathrm{m}$; Aperture: Tri-colporate; Exine: $4 \mu \mathrm{m}$, sexine much thicker than nexine, granulated, alveolate.

\section{Family: Myricaeae}

Myrica sp. (Plate VI, Fig. 65)

Shape: Oblate, triangular in polar view; Size: 18-20 $\mu \mathrm{m}$; Aperture: Tri-cilporate; Exine: $1 \mu \mathrm{m}$, sexine is thicker than nexine near and around pori, sexine microechinate.

\section{Family: Oleaceae}

\section{Fraxinus sp. (Plate VI, Fig. 66)}

Shape: Prolate, circular in polar view; Size: $15 \mu \mathrm{m}$; Aperture: Tri-colporate, colpi as long as polar axis; Exine: $1.5 \mu \mathrm{m}$, sexine thicker than nexine, granulated, alveolated.

\section{Jasminum sp. (Plate VI, Fig. 67)}

Shape: Spheroidal, circular in polar view; Size: $38 \mu \mathrm{m}$; Aperture: Tri-colporate; Exine: $3 \mu \mathrm{m}$, sexine thicker than nexine, surface of the exine is reticulated.

\section{Ligustrum sp. (Plate VI, Fig. 68)}

Shape: Spheroidal to sub-prolate, circular in polar view; Size: $22 \mu \mathrm{m}$; Aperture: Tri-colporate; Exine: $2.5 \mu \mathrm{m}$, sexine thicker than nexine, exine reticulated, foveolated.

\section{Family: Onagraceae}

Onagraceae gen. indet. (Plate VII, Fig. 69)

Shape: Tetrahedral tetrads; Size: 55-60 $\mu \mathrm{m}$; Aperture: Tricolporate; Exine: $5 \mu \mathrm{m}$, sexine comparatively thicker than nexine, aspidated, exine distrinctly stratified, reticulated.

\section{Family: Polygonaceae}

\section{Polygonum posumbo (Plate VII, Fig. 70)}

Shape: Globular; Size: 10-15 $\mu \mathrm{m}$; Aperture: Pantoporate; Exine: $1.5 \mu \mathrm{m}$, sexine thicker than nexine, exine stratified and reticulated.

\section{Polygonum sp. (1) (Plate VII, Fig. 71)}

Shape: Spheroidal; Size: 30-32 $\mu \mathrm{m}$; Aperture: Pantoporate; Exine: $4 \mu \mathrm{m}$, sexine and nexine similar in size, reticulated, alveolated, columella long and closely packed, lumina with numerous rudimentary columellae.

\section{Polygonum sp. (2) (Plate VII, Fig. 72)}

Shape: Spheroidal; Size: 35 m; Aperture: Pantoporate; Exine: $5 \mu \mathrm{m}$, sexine thicker than nexine, granulated, reticulated, micro 


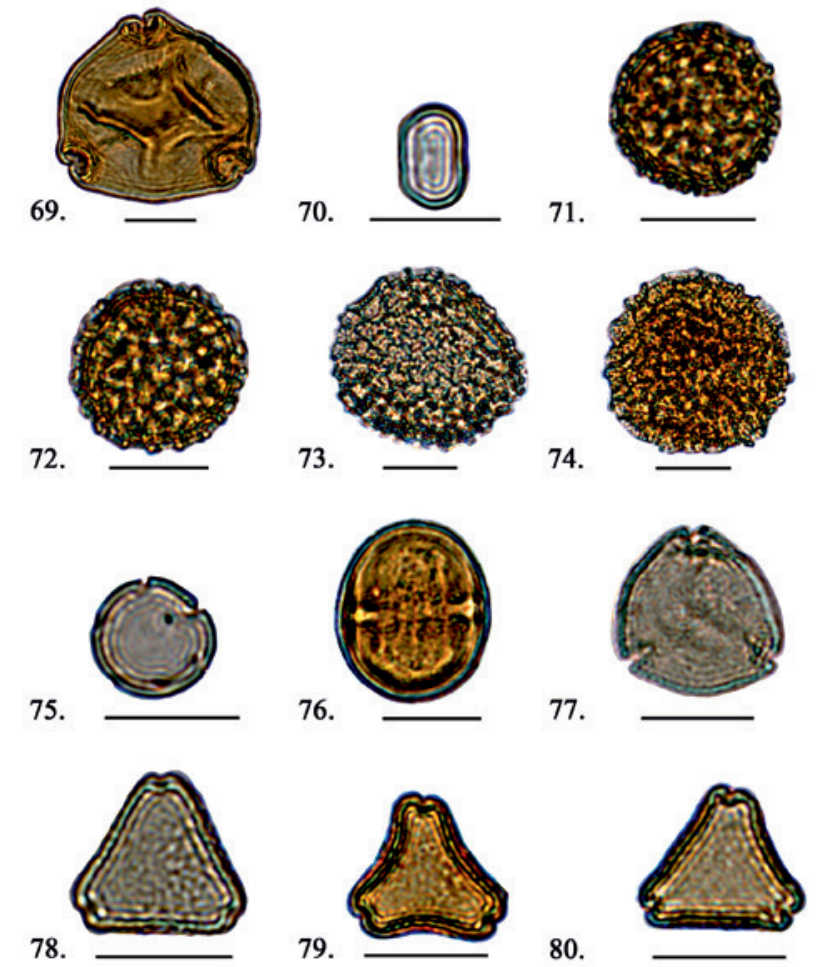

Plate-VII: 69. Family Onagraceae gen. Indet., 70. Polygonum posumbu, 71. Polygonum sp. (1), 72. Polygonum sp. (2), 73. Polygonum sp. (3), 74. Polygonum sp. (4), 75. Family Rubiaceae gen. indet., 76. Family Sapotaceae gen. indet., 77. Symplocos sp. (1), 78. Symplocos sp. (2), 79. Symplocos sp. (3), 80. Symplocos sp. (4) (scale bar $=20 \mu \mathrm{m}$ ).

spinules are seen on the edge, laminated.

Polygonum sp. (3) (Plate VII, Fig. 73)

Shape: Spheroidal; Size: 55-58 $\mu$; Aperture: Pantoporate; Exine: $4 \mu \mathrm{m}$, sexine much thicker than nexine, reticulated, micro spinules, alveolated, columell along and closely packed.

\section{Polygonum sp. (4) (Plate VII, Fig. 74)}

Shape: Spheroidal; Size: 57-60 $\mu \mathrm{m}$; Aperture: Pantoporate; Exine: $5 \mu \mathrm{m}$, sexine thicker than nexine, granulated, reticulated, micro spinules are seen on the edge, laminated.

\section{Family: Rubiaceae}

Rubiaceae gen. indet. (Plate VII, Fig. 75)

Shape: Spheroidal, circular in polar view; Size: 15-17 $\mu$ m; Aperture: Pentaporate; Exine: $3 \mu \mathrm{m}$, sexine thicker than nexine, reticulated, perforated.

\section{Family: Sapotaceae}

Sapotaceae gen. indet. (Plate VII, Fig. 76)

Shape: Prolate, circular in polar view; Size: 28-39 $\mu \mathrm{m}$; Aperture: Tetra-colporate; Exine: $4 \mu \mathrm{m}$, sexine thicker than nexine, perforated, alveolated.
Family: Symplocaceae

Symplocos sp. (1) (Plate VII, Fig. 77)

Shape: Triangular in polar view; Size: 30-32 $\mu \mathrm{m}$; Aperture: Tri-colporate; Exine: $3 \mu \mathrm{m}$, sexine much thicker than nexine, roughly verrucate exine surface.

\section{Symplocos sp. (2) (Plate VII, Fig. 78)}

Shape: Triangular in polar view; Size: 20-22 $\mu$; Aperture: Tri-colporate; Exine: $2 \mu \mathrm{m}$, sexine thicker than nexine, exine stratified, reticulated.

\section{Symplocos sp. (3) (Plate VII, Fig. 79)}

Shape: Triangular in polar view; Size: $22-25 \mu \mathrm{m}$; Aperture: Tricolporate; Exine: $4 \mu \mathrm{m}$, sexine and nexine similar in thickness, exine surface smoothly verrucated, reticulated.

\section{Symplocos sp. (4) (Plate VII, Fig. 80)}

Shape: Triangular in polar view; Size: $25-28 \mu \mathrm{m}$; Aperture: Tri-colporate; Exine: $3 \mu \mathrm{m}$, sexine slightly thicker than nexine, exine stratified.

Family: Ulmaceae

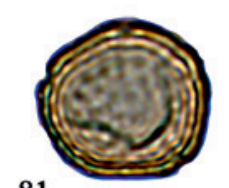

81.

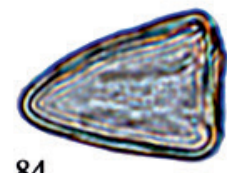

84.

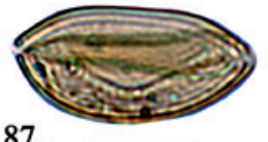

87.

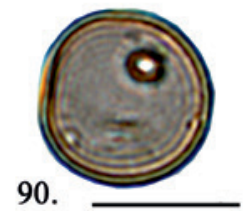

82.

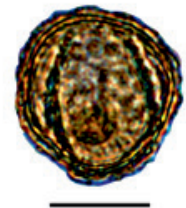

85.
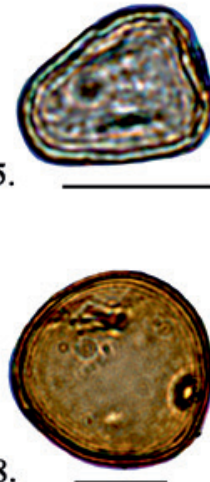

91.

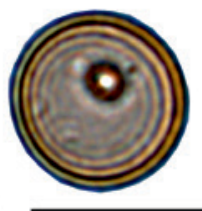

83.

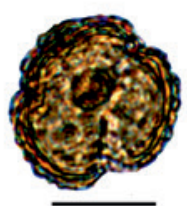

86.

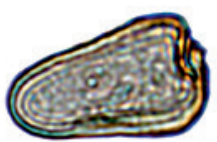

89.

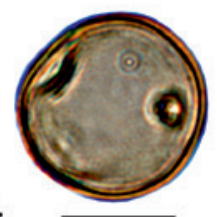

92.

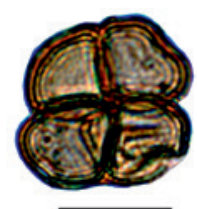

Plate-VIII: 81. Ulmus sp. 82-83. Family Valerianaceae gen. indet. (Equatorial and Polar View) 84. Cyperaceae gen. indet. (1), 85. Cyperaceae gen. Indet. (2), 86. Cyperaceae gen. Indet. (3), 87. Liliaceae gen. indet., 88-91. Poaceae gen. Indet., 92. Typha sp. (scale bar $=20 \mu \mathrm{m})$. 
Ulmus sp. (Plate VIII, Fig. 81)

Shape: Oblate, pentagonal in polar view; Size: $22 \mu \mathrm{m}$; Aperture: Pentaporate; Exine: $1.5 \mu \mathrm{m}$, thicker sexine and regulated, walls are fragile and pores are typically less aspidate.

\section{Family: Valerianaceae}

Valerianaceae gen. indet. (Plate VIII, Figs. 82, 83)

Shape: Spherical in equatorial view, circular in polar view; Size: Equatorial axis $34 \mu \mathrm{m}$, Polar axis: $30 \mu \mathrm{m}$; Aperture: Tricolporate; Exine: $5 \mu \mathrm{m}$, exine slightly thicker, columellae in mesocolpia simple, sparser, irregular in shape, branched above, ornamentation micro-echinate, verrucae absent.

\section{iii. Angiosperms (Monocotyledons)}

\section{Family: Cyperaeae}

Cyperaceae gen. indet. (1) (Plate VIII, Fig. 84)

Shape: Triangular; Size: $36 \mu \mathrm{m}$; Aperture: Monoporate; Exine $2.5 \mu \mathrm{m}$, sexine slightly thicker than nexine, smoothly covered exine, granulated, micro-echinated.

Cyperaceae gen. indet. (2) (Plate VIII Fig. 85)

Shape: Triangular; Size: $25 \mu \mathrm{m}$; Aperture: Monoporate; Exine: $2.8 \mu \mathrm{m}$, Sexine much thicker than nexine, perforated, microechinated.

Cyperaceae gen. indet. (3) (Plate VIII, Fig. 86)

Shape: Triangular; Size: 30-35 $\mu \mathrm{m}$; Aperture: Monoporate; Exine: $2.7 \mu \mathrm{m}$, sexine slightly thicker than nexine, perforated, reticulated.

\section{Family: Liliaceae}

Liliaceae gen. indet. (Plate VIII, Fig. 87)

Shape: Oblate, elliptic; Size: $40-44 \mu \mathrm{m}$; Aperture: Monosulcate; Exine: scabrated, reticulum irregular, very small lumina present.

\section{Family: Poaceae}

Poaceae gen. indet. (1) (Plate VIII, Fig. 88)

Shape: Spherical; Size: $40 \mu \mathrm{m}$; Aperture: Monoporate; Exine: $2 \mu \mathrm{m}$, sexine and nexine similar in thickness, micro-echinate, perforated.

Poaceae gen. indet. (2) (Plate VIII, Fig. 89)

Shape: Spherical; Size: 32-34 $\mu \mathrm{m}$; Aperture: Monoporate; Exine: $1.5 \mu \mathrm{m}$, sexine and nexine similar in thickness, microechinate, perforated.

Poaceae gen. indet. (3) (Plate VIII, Fig. 90)

Shape: Spherical; Size: $25 \mu \mathrm{m}$; Aperture: Monoporate; Exine: $2 \mu \mathrm{m}$, stratified and smooth, both sexine and nexine similar.
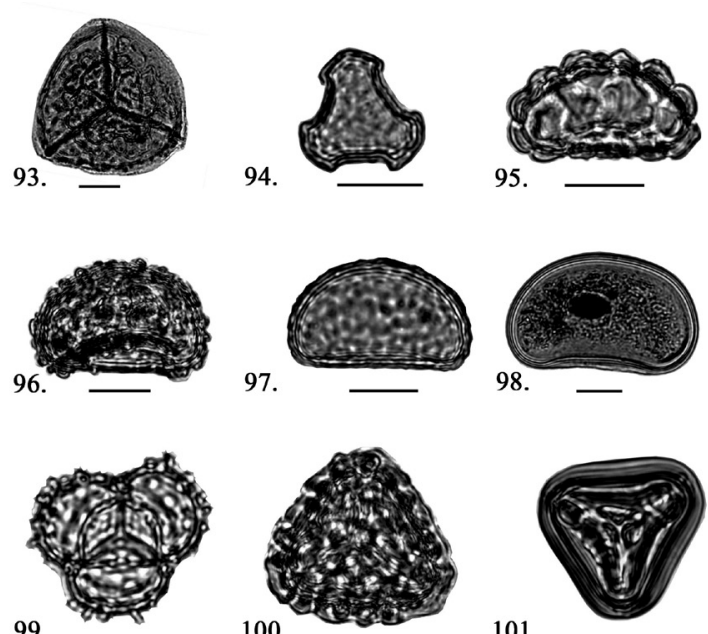

99.
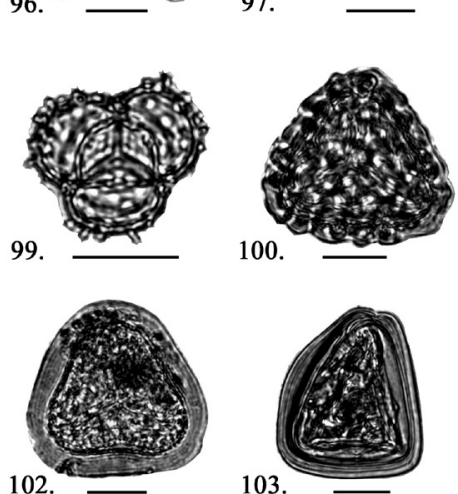

101
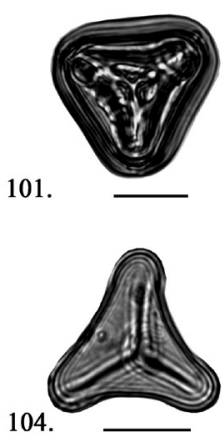

Plate-IX: 93. Lycopodium sp. (1), 94. Lycopodium sp. (2) 95. Arthromeris sp. 96. Polypodium sp. 97. Davallia sp. 98. Polypodium sp. 99. Selaginella sp. 100. Pteris sp. (1) 101. Pteris sp. (2), 102. Pteris sp. (3), 103. Pteris sp. (4), 104. Cyathea sp. (scale bar $=20 \mu \mathrm{m})$.

\section{iv. Pteridophytes}

Family: Lycopodiaceae

Lycopodium sp. (1) (Plate IX, Fig. 93)

Shape: Semi-circular in polar view; Size: $75-80 \mu \mathrm{m}$; Aperture: Trilete.

Lycopodium sp. (2) (Plate IX, Fig 94)

Shape: Triangular; Size: 25-30 $\mu \mathrm{m}$; Aperture: Trilete.

\section{Family: Polypodiaceae}

Arthromeris sp. (Plate IX, Fig. 95)

Shape: Bilateral in polar view and kidney shaped in equatorial view; Size: $45 \mu \mathrm{m}$; Aperture: Monolete; Exospore: $5 \mu \mathrm{m}$, spinose and granulose, thickly undulated.

\section{Polypodium sp. (Plate IX, Fig. 96)}

Shape: Bilateral; Size: $90 \mu$ m; Aperture: Monolete; Exospore: $5 \mu \mathrm{m}$, spinose, roughly granulate.

\section{Family: Selaginellaceae}

Selaginella sp. (Plate IX, Fig. 99)

Shape: Circular, lobate; Size: 30-32 $\mu \mathrm{m}$. Aperture: Trilete; Exospore: $3 \mu \mathrm{m}$, roughly echinated, slightly rugulated. 


\section{Family: Pteridaceae}

Pteris sp. (1) (Plate IX, Fig. 100)

Shape: Triangular in polar view, straight to concave side and rounded corners; Size: 45-50 $\mu \mathrm{m}$; Aperture: Trilete; Exospore is proximally verrucate and distally regulate.

Pteris sp. (2) (Plate IX, Fig. 101)

Shape: Triangular in polar view; Size: 40-45 $\mu \mathrm{m}$; Aperture: Trilete; Verrucated exospores and distally regulate.

Pteris sp. (3) (Plate IX, Fig. 102)

Shape: Circular, Triangular in polar view; Size: 50-55 $\mu \mathrm{m}$; Aperture: Trilete; Exospore with rounded corners, $5 \mu \mathrm{m}$, roughly granulose.

Pteris sp. (4) (Plate IX, Fig. 103)

Shape: Triangular in polar view; Size: 40-42 $\mu \mathrm{m}$; Aperture: Trilete; Exospore slightly thicker than endospore, distally rugulated and proximally verrucate.

\section{Family: Cyatheaceae}

\section{Cyathea sp. (Plate IX, Fig. 104)}

Shape: Triangular, concave sides and rounded corners; Size: 35 $\mu \mathrm{m}$; Aperture: Trilete. The exposure is $1.2-3 \mu \mathrm{m}$ thick, psilate, perforate or verructae.

\section{POLLEN ASSEMBLAGE ZONES}

The pollen diagram prepared on the basis of palynomorphs shows three pollen assemblage zones; i.e. P-I, P-II and P-III in ascending order (Fig. 3).

\section{Zone P - I}

The ZONE P-I is the lowermost zone of this section and is featured by the dominance of Pinus (12.3-32.12\%) followed by Picea (0.3-1.08\%), Tsuga (0.43-1.71\%) and very few
Podocarpus. Similarly, angiosperm trees like and Quercus $(8.59-22.46 \%)$ have the dominance in their group. Herbs like Poaceae (8.03-26.04\%) are high and Artemisia, Compositae and Caryophyllaceae are smaller in percentages. Pteridophytes are represented by Polypodiaceae (1.56-23.91\%), Triletes (1.28$8.85 \%$ ) and other Monoletes (1.2-4.81\%). Wetland plants are represented by Polygonaceae, Cyperaceae and Liliaceae.

\section{Zone P-II}

This zone shows the significant decrease of pollen compared to previous zone. Gymnosperm such as Pinus (19.46-24.88\%) has declined, whereas Picea (4-5.88\%) and Podocarpus $(0.67-2 \%)$ have retained their presence in similar way. The most distinguishing feature of this zone is the presence of Cedrus, which is completely absent in other zones. Quercus and Poaceaewhich were dominant in Zone P-I has declined in significant number. Angiosperms like Alnus and Betula shows weak presence whereas species like Castanopsis, Juglan, Myrica, Fraxinus are almost absent. Artemisia is most common among herbs. The wetland plants belonging to families Cyperaceae, Polygonaceae, and Liliaceae are dominant as in the previous pollen assemblage zone. Among Pteridophytes, Polypodiaceae (2.26-11.16\%) and other Triletes (4.03-6\%) are common.

\section{Zone P-III}

The Zone P-III is witnessed by the heavy increase of Quercus (12.14-24.06\%). Similarly the percentage of gymnosperms such as Picea (3.26-10.5\%), Pinus and Tsuga (3.27-7.34\%) increased. Alnus and Betula also show their significant presence in this zone. Artemisia and other Compositae are evident. The percentage of Poaceae is fluctuating between $5-10 \%$ of the total pollen sum. Among the wetland plants Polygonum and Cyperaceae are still prominent while Liliaceae decreased. In Pteridophytes Polypodiaceae is dominant (3.84-21.79\%) which is followed by Pteridaceae (0.75-3.98\%) and other Triletes (0.66-14.68\%). The percentage of Polypodiaceae has gradually increased towards the top.

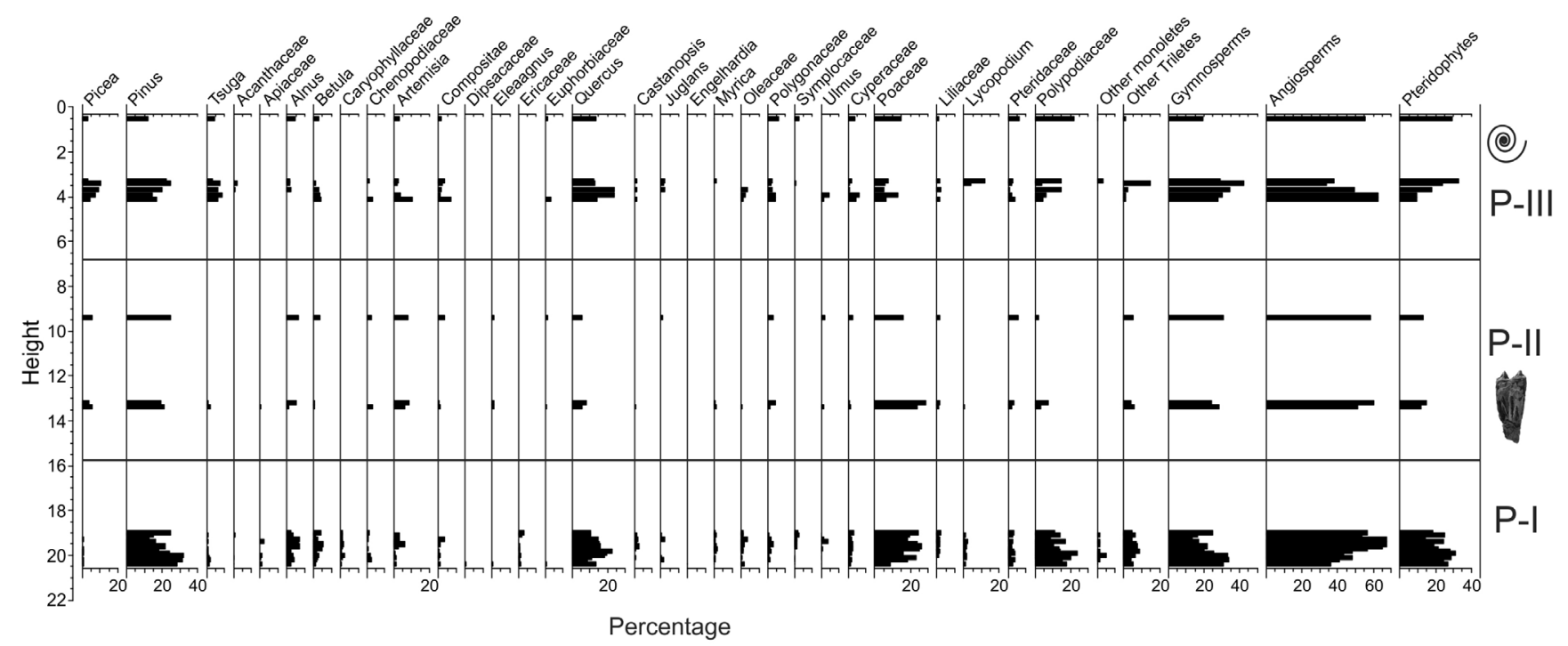

Fig. 3: Pollen diagram of the Thimi Formation, Phaidhoka section, Bhaktapur. 


\section{PALEOCLIMATE}

The pollen analysis from the Phaidhoka Section of the Thimi Formation reveals three major pollen zones i.e. P-I, P-II and P-III. Every pollen assemblages were compared to present vegetation of central Nepal assuming that living plants had similar requirement of climate conditions compared to those of modern ones. Different plants carry their peculiar climatic signatures. Gymnosperms like Pinus represents sub-tropical to cool temperate climate whereas Picea represents cold and dry climate. Similarly, Tsuga represents moist cool temperate areas with high rainfall, cool summers and little or no water stress while major angiosperms such as Quercus represents sub-tropical to cold temperate climate. Similarly Alnus and Betula are found in temperate zones. Artemisia grows in temperate climates of both hemispheres, usually in dry or semiarid habitats. Compositae is most common in the arid and semiarid regions of subtropical and lowers temperate latitudes. Polypodiaceae and Pteridaceae are found in wet climates, most commonly in wet forest floor, representing humid climate. Polygonaceae, Cyperaceae, Typhaceae and Haloragaceae grow near water resources along the bank of river, pond and lakes.

In the pollen assemblage P-I, the dominant species among gymnosperm was Pinus, Other gymnosperms like Picea and Tsuga are present in minor numbers. The angiosperm tree Quercus is most dominant throughout the zone following by Betula indicating cold climate. Alnus indicate the wet condition in riverine and shadow areas. Grass pollen belonging to Poaceae are also dominant indication much dry condition. Pteridophytes fern like Polypodicaeae are found in wet regions. So the vegetation pattern of this assemblages if not uniform, but the majority of vegetation indicates the assemblage as warm temperate climate.

The pollen assemblage P-II marks as the significant decrease of pollen counts. Presence of frequent sandy layers made palynological sampling difficult in this part of the lithological section. Clastic nature of sediments may be responsible for yielding less pollen concentration. Picea has increased compared to previous pollen assemblage zone whereas Pinus, Alnus, Betula, Quercus, Poaceae and Polypodiaceae are thinly distributed. From the vegetation pattern, the climate can be concluded as colder than the previous pollen assemblage zone.

The pollen assemblage P-III marks the increment of Pinus which is dominant among Gymnosperms. Picea and Tsuga are dominant indicating much colder than zone P-II. Angiosperm tree Quercus and Juglans have increased indicating much colder in the upper part of this section. The gap in palynological record is evident in this zone because the sediments towards to are more clastic and followed by oxidation which subsequently unfavourable for the pollen preservation. The presence of lignite in upper part of this section indicates swampy and marshy depositional environment. The opercula are of Bithyniidae (Digoniostoma sp. or/and Gabbia sp.) are abundant in this lignite layer. Such kinds of freshwater molluscs live in shallow water on macrophytes and also on muddy substrate of temporary water bodies. The accumulation of empty shells and large number of opercula may be possible in limnic zone of lakes by water transportation and sedimentation over several meters. In every case this water body might be stagnant with large shallow regions. The large bovid molars recorded from the middle part of the section confirms the earlier findings by Paudayal et al. (2011) few hundred meters east of this section.

\section{CONCLUSIONS}

Palynological investigation from the Phaidhoka Section of the Thimi Formation revealed 94 species of plants belonging to 74 genera and 40 families. Palynological record suggests that the climate in the eastern Bhaktapur area was variable and not uniform in Late Quaternary Period. The gymnosperm like Pinus, Picea and Tsuga are most commonhaving higher frequency in middle and upper part of the section. Among the angiosperm trees Quercus, Alnus, Betula, Myrica, Oleaceae and Ericaceae are common. The climate during the deposition of uppermost and middle part of this section was comparatively colder than present day indicating cold temperate signature. However, the pollen assemblages indicate warm temperate climatic condition existed during the deposition of the lowermost part of this section.

\section{ACKNOWLEDGEMENT}

We would like to express our sincere thanks to Prof. Dr. Lalu Paudel, Head of the Central Department of Geology, Tribhuvan University (CDG, TU) Kirtipur, for his support and cooperation to carry out this research at $\mathrm{CDG}$, TU.

\section{REFERENCES}

Bhandari, S. and Paudayal, K. N., 2007, Palynostratigraphy and palaeoclimatic interpretation of the Plio-Pleistocene Lukundol Formation from the Kathmandu valley, Nepal. Jour. Nepal Geol. Soc., v. 35, pp. 1-10.

Ferguson, D. K., Zetter, R., and Paudayal, K. N., 2007, The need for the SEM in Palaeopalynology. Comptes Rendus Palevol, v. 6(6-7), pp. 423-430.

Fuji, R. and Sakai, H., 2001, Palynological study of the drilled sediments from the Kathmandu basin and its palaeoclimatic and sedimentological significance. Jour. Nepal Geol. Soc., v. 25 (Sp. Issue), pp. 53-61.

Fujii, R. and Sakai, H., 2002, Paleoclimatic changes during the last 2.5 myr recorded in the Kathmandu basin, Central Nepal Himalayas. Jour. Asian Earth Sci., v. 20, pp. 255266.

Gajurel, A. Huyghe, P., France-Lanord, C., Mugnier, J. L, Upreti, B. N., and Le Fort, P., 1998, Seismites in Kathmandu basin, in Nepal. Jour. Nepal Geol. Soc., v. 18, pp. 125-134.

Igarashi, Y., Yoshida, M., and Tabata, H., 1988, History of vegetation and climate in the Kathmandu valley. Proc. Indian Nat. Sci. Acad., v. 54A(4), pp. 550-563. 
Kizaki, K., 1994, An Outline of the Himalayan Upheaval, a case study of the Nepal Himalaya. JICA (Japan Intl. Coop. Agency), 127 p.

Moribayashi, S. and Maruo, Y., 1980, Basement topography of the Kathmandu Valley, Nepal- an application of gravitational method to the survey of a tectonic basin in the Himalayas. Jour. Japan Soc. Engg. Geol., v. 21, pp. 30-37.

Paudayal, K. N., 2005, Late Pleistocene pollen assemblages from the Thimi Formation, Kathmandu valley, Nepal. The Island Arc, v. 14(4), pp. 328-337.

Paudayal, K. N., 2006, Late Pleistocene pollen assemblages from the Gokarna Formation, Kathmandu valley. Jour. Nepal Geol. Soc., v. 33, pp. 33-38.

Paudayal, K. N., 2011a, High resolution palynostratigraphy and climate from the Late Quaternary Besigaon section belonging to Gokarna Formation in the Kathmandu valley. Jour. Strat. Assoc. Nepal, v. 7, pp. 33-38.

Paudayal, K. N., 2011b, Palynological assemblages from the Late Pleistocene sediments of the Patan Formation in Kathmandu valley and their climatic implications. Bull. Dept. Geol., Tribhuvan University, v. 14, pp. 59-66.
Paudayal, K. N. and Ferguson, D. K., 2004, Pleistocene palynology of Nepal. Quaternary International, v. 117, pp. 69-79.

Paudayal, K. N., Panthee, S., and Hertler, C., 2011, A bovid specimen from Late Pleistocene deposits in the Kathmandu basin, Nepal. Jour. Strat. Assoc. Nepal, v. 7, pp. 9-14.

Vishnu-Mittre and Sharma, C., 1984, Vegetation and climate during the last glaciation in the Kathmandu valley. Pollen et Spores, v. 36, pp. 69-94.

Yoshida, M. and Gautam, P., 1988, Magnetostratigraphy of Plio-Pleistocene lacustrine deposits in the Kathmandu valley, central Nepal. Proc. Ind. Nat. Sci. Acad., v. 54A(30), pp. 410-417.

Yoshida, M. and Igarashi, Y., 1984, Neogene to Quaternary lacustrine sediments in the Kathmandu valley, Nepal. Jour. Nepal Geol. Soc., v. 4, pp. 73-100.

Zetter, R., 1989, Methodik und bedeutung einer routinemaessig kombinierten lichtmikroskopischen und rasterelektronmikroskopischen untersuchung fossiler mikrofloren. Courier Forschungsinstitut Senckenberg, Frankfurt am Main, v. 109, pp. 41-50. 